\title{
Contrast Preserving Decolorization
}

\author{
Cewu Lu Li Xu Jiaya Jia \\ Department of Computer Science and Engineering \\ The Chinese University of Hong Kong \\ \{cwlu,xuli, leojia\}@cse.cuhk.edu.hk
}

\begin{abstract}
Decolorization - the process to transform a color image to a grayscale one - is a basic tool in digital printing, stylized black-and-white photography, and in many single channel image processing applications. In this paper, we propose an optimization approach aiming at maximally preserving the original color contrast. Our main contribution is to alleviate a strict order constraint for color mapping based on human vision system, which enables the employment of a bimodal distribution to constrain spatial pixel difference and allows for automatic selection of suitable gray scale in order to preserve the original contrast. Both the quantitative and qualitative evaluation bears out the effectiveness of the proposed method.
\end{abstract}

\section{Introduction}

Grayscale is one of the widely used pictorial expressions in digital printing and photograph rendering. Color-to-gray conversion is required in many single-channel image processing applications. Naturally, this type of conversion is a task of dimension reduction, which inevitably suffers from information loss. The general goal is thus to use the limited range in gray scales to preserve as much as possible the original color contrast. It is found that intuitive methods, such as extracting the lightness channel in the CIELab color space [18, 5], would easily diminish salient chromatic structures and lose important appearance features. One example is shown in Fig. 1(a) and (b).

To preserve color contrast, recent color-to-gray methods imposed constraints on spatial intensity variation and required that the grayscale contrast is similar to that of the color input. In general, signed color difference, as well as the color order, for neighboring pixels is specified $[6,9]$ in decolorization based on the Euclidian color distance in the CIELab space. With this stringent constraint, selection of intensity values could lack freedom and suffer from contrast loss. Fig. 1(c)-(e) shows the results of state-of-the-art color-to-gray methods $[6,19,9]$. While details are mostly

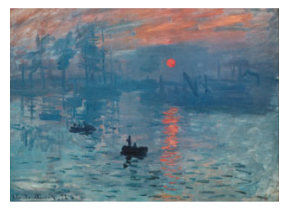

(a) Input

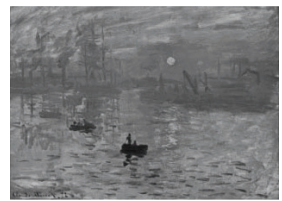

(d) [6]

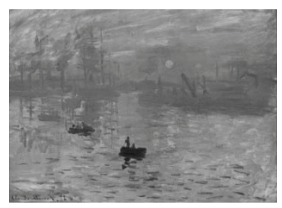

(b) $\mathrm{L}$ of Lab

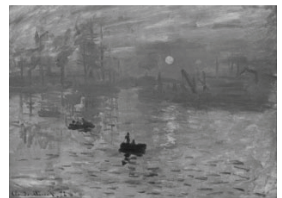

(e) [9]

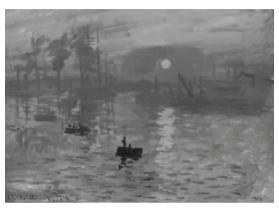

(c) [19]

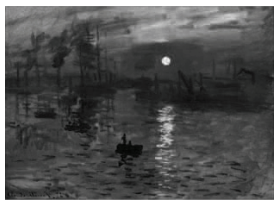

(f) Ours
Figure 1. Color-to-gray conversion. We propose a new method to preserve contrast with respect to the original color image.

preserved, visually conspicuous color change between the sun and sky is not well represented.

In fact, human visual system does not accurately perceive chrominance and lightness. Instead, their relationship to the adjacent context $[13,4]$ plays a vital role. The order of different colors [21] also cannot be defined uniquely by people, as evidenced in psychology and medical research. An example is that the lightness channel in the CIELab color system makes green brighter than blue. But recent study indicates that people with different culture and language background have different senses of brightness with respect to color. Someone feel just the opposite [16, 23].

Based on these conclusions, we relax the color order constraint and present a new method seeking better preservation of color contrast and significant enhancement of visual distinctiveness for edges. These benefits stem from the new weak color order constraint, which allows for a very flexible and practical color-to-gray model. For color pairs without a clear order in brightness, we propose a bimodal distribution, i.e., mixture of two Gaussians, to automatically find suitable orders with respect to the visual context in optimization.

Our other main contributions include designing a parameterized multivariate polynomial function for color mapping 
and developing a fixed point iteration solver for the novel energy function. We also propose a new quantitative metric to numerically compare the performance of contrast preservation, from a human perceptual point of view. We extensively evaluate our method on a large number of synthetic and natural images.

\section{Related Work}

Decolorization can be performed either locally or globally. Local methods process pixels differently, and usually rely on local chrominance edges for enhancement. Bala and Eschbach [1] added high frequency components of chromaticity to the lightness channel, in order to enhance color edges. Neumann et al. [15] locally selected consistent color gradients and performed fast 2D integration to get the final grayscale image. Smith et al. [19] also employed a local sharpening step after obtaining the grayscale image by global mapping. Chrominance edges are enhanced by adaptively-weighted multi-scale unsharp masking. These mechanisms might occasionally distort the appearance of constant color regions and produce visual artifacts, as discussed in [9].

In global mapping, Gooch et al. [6] enforced color contrast between pixel pairs. Rasche et al. [17] defined constraints directly on different color pairs. A linear color mapping is adopted for acceleration. Kuk et al. [10] extended the idea of [6] by considering both the global and local contrasts. Grundland and Dodgson [7] proposed a fast linear mapping algorithm that adds a fixed amount of chrominance to the lightness, where the original lightness and color order can be better preserved by restraining the added chrominance. A parametric piecewise linear mapping is used to convert color to gray.

Kim et al. [9] proposed a non-linear parametric model for color-to-gray mapping. The parameters are estimated by minimizing the cost function that aims to preserve color difference computed in the CIELab color space. Song et al. [20] incorporated three visual cues in a global energy function, optimized using a variational approach. Lee et al. [12] performed decolorization by adding contrast information back to luminance. Lau et al. [11] defined their energy function on a clustered color image. This method is able to perform transformation between different color spaces.

In short, to preserve color contrast, most of the previous methods explicitly specify color order. This strategy may shrink the space for optimally picking grayscale values and lead to less optimal solutions in terms of retaining originally prominent contrast.

\section{Parametric Color-to-Gray Model}

Our decolorization function is defined as $g=f(\mathbf{c})$. For each input RGB vector $\mathbf{c}=(r, g, b)$, function $f$ pro-

\begin{tabular}{|c|c|c|}
\hline & mean & variance \\
\hline \hline Nayatani model & $1.28 \times 10^{-2}$ & $2.61 \times 10^{-3}$ \\
L channel of Lab & $3.20 \times 10^{-3}$ & $8.71 \times 10^{-3}$ \\
Y channel of YUV & 0 & 0 \\
\hline
\end{tabular}

Table 1. Mean and variance of the fitting errors when using our method to approximate other widely adopted models. All gray levels are mapped into range $[0,1]$.

duces $g$, the corresponding gray-scale value. We adopt a global mapping scheme where all color pixels in the input are converted to grayscale using the same mapping function $f$. Therefore, two pixels with the same color will have the same gray scale.

In our method, we adopt a finite multivariate polynomial function for mapping. Mathematically, we define the polynomial space of color $\mathbf{c}$ with its degree $n$ as

$\Pi_{n}=\operatorname{span}\left\{r^{d_{1}} g^{d_{2}} b^{d_{3}}: d_{i}=0,1,2, \ldots, d_{1}+d_{2}+d_{3} \leq n\right\}$,

where $\Pi_{n}$ is a polynomial space spanned by a family of monomials. The mapping function is thus expressed as

$$
f(r, g, b ; \omega)=\sum_{i} \omega_{i} m_{i}
$$

where $m_{i}$ is the $i$ th monomial basis of $\Pi_{n}$. The mapping function is uniquely determined by weights $\left\{\omega_{i}\right\}$. Empirically, we use degree $n=2$, which means the total number of $\{\omega\}$ is 9 and the mapping function is a linear combination of elements in $\left\{r, g, b, r g, r b, g b, r^{2}, g^{2}, b^{2}\right\}$.

We note that the polynomial form is actually a generalization of common linear and nonlinear color-to-gray mapping functions. To verify it, we experiment with the image set [2], which contains 24 color images. We first generate grayscale images using common methods, including the lightness of CIELab [8], intensity in the YUV space $[18,5]$, and the Nayatani model [14]; the last one is known as highly nonlinear. We then fit our model to approximate these grayscale images using quadratic regression, i.e., by minimizing $\left\|f(\mathbf{c}, \omega)-g^{\prime}\right\|^{2}$, where $g^{\prime}$ denotes the grayscale results. Table 3 lists the fitting errors. They are all very small, indicating that our parametric model is capable to work the same way as these color-to-gray linear and nonlinear mappings.

\section{Bimodal Contrast-Preserving Objective Function}

We describe in this section our color contrast preserving objective function based on a weak color order constraint. To begin with, We revisit the energy used in previous approaches for contrast preserving decolorization. The gray scales for pixels $x$ and $y$, denoted by $g_{x}$ and $g_{y}$ respectively, 
are estimated by minimizing energy function

$$
\min _{g} \sum_{x, y}\left(g_{x}-g_{y}-\delta_{x, y}\right)^{2}
$$

where the result $g$ could be with [9] or without [6] a parametric form. $x$ and $y$ index an ordered pixel pair. $\delta_{x, y}$ is the color contrast, having a signed value indicating the difference of a color pair. Based on the Euclidian distance in the CIELab color space, color contrast is generally expressed as

$$
\left|\delta_{x, y}\right|=\sqrt{\left(L_{x}-L_{y}\right)^{2}+\left(a_{x}-a_{y}\right)^{2}+\left(b_{x}-b_{y}\right)^{2}},
$$

which represents the color dissimilarity in the human vision system [22]. The sign of $\delta_{x, y}$ is typically determined by the $\operatorname{sign}$ in the $L$ channel, i.e. $\operatorname{sign}\left(L_{x}-L_{y}\right)$. As discussed in Section 1, enforcing this type of order for color pairs could cause the contrast-loss problem. Also, it may not be in obedience to human visual perception, where orders are ambiguous. In our method, the condition is loosen by encouraging a bimodal selection mechanism.

Eq. (3) can be interpreted in view of probabilistic inference. It implies that the grayscale difference of two pixels $x$ and $y$ follows a Gaussian distribution with mean $\delta_{x, y}$. Minimizing Eq. (3) is thus equivalent to maximizing likelihood

$$
\prod_{x, y} G\left(\delta_{x, y}, \sigma^{2}\right) \propto \prod_{x, y} \exp \left\{-\frac{\left|\Delta g_{x, y}-\delta_{x, y}\right|^{2}}{2 \sigma^{2}}\right\} .
$$

The Gaussian distribution has a single mode peaked at $\delta_{x, y}$, which implies not only the contrast is constrained, but also the sign of gray-level difference is determined. It is noteworthy that the sign does not embody an exact physical meaning. So the difference is allowed to be either $+\delta_{x, y}$ or $-\delta_{x, y}$, which gives rise to a flexible and proper contrast preserving constraint. This motivates our work, which uses a bimodal distribution to automatically select color orders.

Weak Color Order Some color pairs can be clearly ordered in terms of brightness. For example, absolute white is always brighter than other colors in common sense. For these color pairs, a single-peak distribution like the one in Eq. (4) is a natural choice, indicating an unambiguous prior. We treat color pairs that satisfy the following constraint as unwavering:

$$
\mathbf{c}_{x} \leq \mathbf{c}_{y} \Longleftrightarrow r_{x} \leq r_{y} \& g_{x} \leq g_{y} \& b_{x} \leq b_{y} .
$$

If Eq. (5) is satisfied, the sign of $\delta_{x, y}$ directly applies to $g_{x}-g_{y}$. Otherwise, we do not specify the sign in prior but instead propose a selection procedure to optimally find the suitable color order. Our likelihood term for one pixel pair is therefore defined as

$$
\frac{1}{2}\left\{G\left(\delta_{x, y}, \sigma^{2}\right)+G\left(-\delta_{x, y}, \sigma^{2}\right)\right\}
$$

Given the above two types of order definition, we build a map to distinguish them. It is constructed as

$$
\alpha_{x, y}= \begin{cases}1.0 & \text { if } r_{x} \leq r_{y}, g_{x} \leq g_{y}, b_{x} \leq b_{y} \\ 0.5 & \text { otherwise }\end{cases}
$$

If $\alpha_{x, y}=1$, we apply prior $G\left(\delta_{x, y}, \sigma^{2}\right)$ for unambiguous color order enforcement. Otherwise, we let the color difference follow a bimodal distribution, which allows for selection of the positive or negative sign optimally. The final objective function is written as

$$
\prod_{(x, y) \in \mathcal{N}}\left\{\alpha_{x, y} G\left(\delta_{x, y}, \sigma^{2}\right)+\left(1-\alpha_{x, y}\right) G\left(-\delta_{x, y}, \sigma^{2}\right)\right\} .
$$

$(x, y) \in \mathcal{N}$, where $\mathcal{N}$ is the four-neighbor set.

Maximizing Eq. (8) is equivalent to minimizing its negative logarithm, expressed as

$$
E(g)=-\sum_{(x, y) \in \mathcal{N}} \ln \left\{\alpha_{x, y} G\left(\delta_{x, y}, \sigma^{2}\right)+\left(1-\alpha_{x, y}\right) G\left(-\delta_{x, y}, \sigma^{2}\right)\right\}
$$

Substituting in Eq. (9) the parametric gray model described in Section 3, a function is formed consisting of unknown coefficients $\left\{\omega_{i}\right\}$. As the global non-linear mapping is used, only nine parameters need to be estimated.

The difference of two gray pixels can then be expressed with respect to the parameters $\left\{\omega_{i}\right\}$ :

$$
\begin{aligned}
\Delta g_{x, y} & =g_{x}-g_{y} \\
& =f\left(r_{x}, g_{x}, b_{x}\right)-f\left(r_{y}, g_{y}, b_{y}\right) \\
& =\sum_{i} \omega_{i}\left(m_{i x}-m_{i y}\right) .
\end{aligned}
$$

We further denote $l_{i(x, y)}:=m_{i x}-m_{i y}$, which can be directly computed given the color of pixels $x$ and $y$. The energy function w.r.t. parameter set $\omega$ is finally written as

$$
\begin{array}{r}
E(\omega)=-\sum_{(x, y) \in \mathcal{N}} \ln \left\{\alpha_{x, y} \exp \left\{-\frac{\left|\sum_{i} \omega_{i} l_{i(x, y)}-\delta_{x, y}\right|^{2}}{2 \sigma^{2}}\right\}+\right. \\
\left.\left(1-\alpha_{x, y}\right) \exp \left\{-\frac{\left|\sum_{i} \omega_{i} l_{i(x, y)}+\delta_{x, y}\right|^{2}}{2 \sigma^{2}}\right\}\right\} .
\end{array}
$$

We describe in the next section the numerical solver.

\section{Numerical Solution}

In the energy function (11), taking partial derivatives with respect to $\left\{\omega_{i}\right\}$ and setting them to zeros yield an equation system. To simplify presentation, we define

$$
\beta_{x, y}:=\frac{\alpha_{x, y} G\left(\delta_{x, y}, \sigma^{2}\right)}{\alpha_{x, y} G\left(\delta_{x, y}, \sigma^{2}\right)+\left(1-\alpha_{x, y}\right) G\left(-\delta_{x, y}, \sigma^{2}\right)} .
$$

With a few algebraic operations, the partial derivative on $\omega_{j}$, i.e. $\frac{\partial E(\omega)}{\partial \omega_{j}}$, can be expressed as

$$
\sum_{(x, y) \in \mathcal{N}} \sum_{i} \omega_{i} l_{i(x, y)} l_{j(x, y)}+\left(1-2 \beta_{x, y}\right) l_{j(x, y)} \delta_{x, y}=0 .
$$




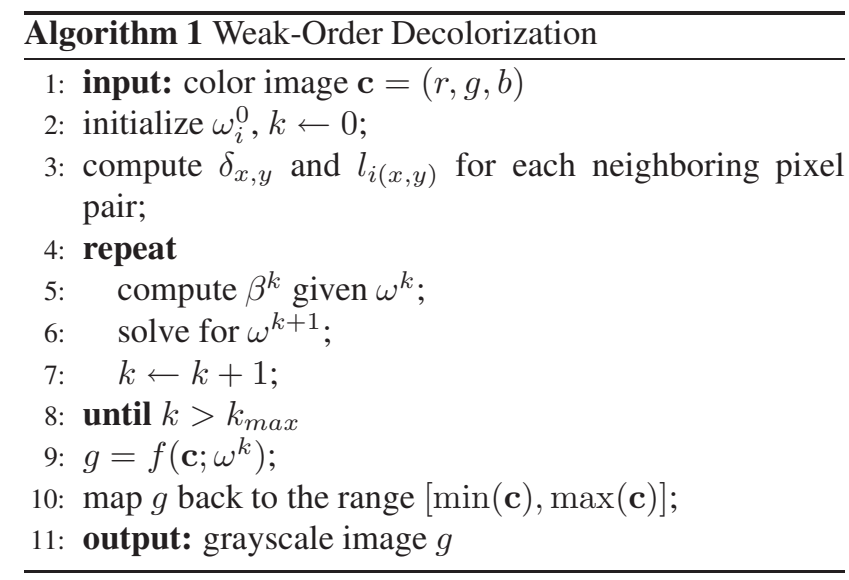

By setting $\frac{\partial E(\omega)}{\partial \omega_{j}}$ to zeros, we obtain a total of 9 equations. The difficulty in solving it stems from the terms $\beta_{x, y}$, which contain nonlinear functions about $\omega$. We apply the fix-point iteration strategy on $\omega$ to linearize the corresponding equations. Specifically, to solve for $\omega_{i}^{k+1}$ in the $k+1$ th iteration, we use the previously estimated $\omega_{i}^{k}$ to generate the nonlinear term $\beta_{x, y}^{k}$, which yields equations

$$
\sum_{(x, y) \in \mathcal{N}} \sum_{i} \omega_{i}^{k+1} l_{i(x, y)} l_{j(x, y)}=\left(2 \beta_{x, y}^{k}-1\right) l_{j(x, y)} \delta_{x, y} .
$$

Now the unknowns only exist in the left hand side of Eq. (5) and we have 9 equations in the form of Eq. (5) by varying $j$. In each iteration, the system is linear w.r.t. $\left\{\omega_{i}^{k+1}\right\}$ and thus can be solved easily. In implementation, we use the backslash operator " " in Matlab.

Our computation framework is sketched in Algorithm 1. We first initialize $\left\{\omega_{i}^{0}\right\}$ as $\{0.33,0.33,0.33,0,0,0,0,0,0\}$, for simplicity's sake. Then the weights are iteratively updated. The maximum number of iterations $k_{\max }$ is set to 15 empirically. Fig. 2 shows an example where the grayscale image is updated in iterations. The corresponding 9 coefficients $\left\{\omega_{i}\right\}$ are listed in Table 2 . It converges quickly. To make the resulting grayscale image $g$ viewable, we linearly scale values with respect to the largest and smallest values $\max (\mathbf{c})$ and $\min (\mathbf{c})$ in the original color image. To process a $600 \times 600$ color image, our Matlab implementation takes 0.8 s on a desktop PC equipped with an Intel Core i7-2600 CPU. The source code is downloadable from our project website ${ }^{1}$.

\section{Experimental Results}

We compare our method with state-of-the-arts [6, 19, 9]. Note that the method of [19] combines global and local operations; in [6] and [9], global methods are employed with and without a parametric color-to-gray mapping function.

\footnotetext{
${ }^{1}$ http://www.cse.cuhk.edu.hk/\% 7eleojia/projects/color2gray
}

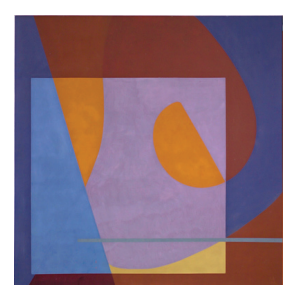

Input

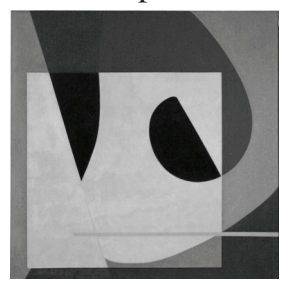

Iteration 3

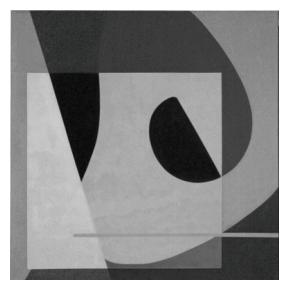

Iteration 13

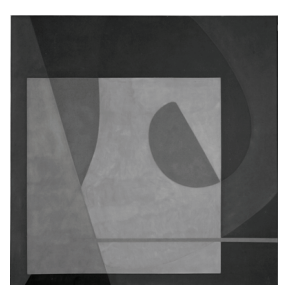

Iteration 1

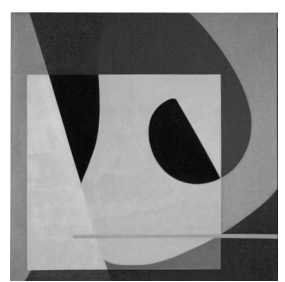

Iteration 4

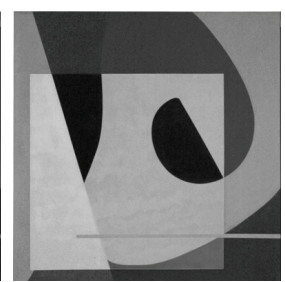

Iteration 14

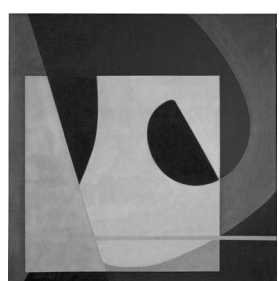

Iteration 2

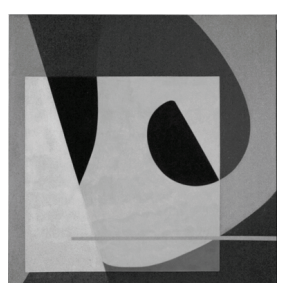

Iteration 5

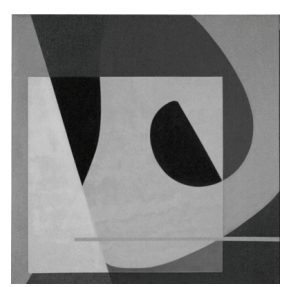

Iteration 15
Figure 2. Results in iterations.

\begin{tabular}{|c||c|c|c|c|c|c|c|c|c|}
\hline iter. & $r$ & $g$ & $b$ & $r g$ & $r b$ & $g b$ & $r^{2}$ & $g^{2}$ & $b^{2}$ \\
\hline \hline 1 & 0.33 & 0.33 & 0.33 & 0.00 & 0.00 & 0.00 & 0.00 & 0.00 & 0.00 \\
\hline 2 & 0.97 & 0.91 & 0.38 & -3.71 & 2.46 & -4.01 & -0.42 & 4.00 & 0.79 \\
\hline 3 & 1.14 & -0.25 & 1.22 & -1.55 & 1.53 & -3.51 & -1.18 & 3.32 & 0.69 \\
\hline 4 & 1.33 & -1.61 & 2.10 & 1.35 & -0.36 & -1.61 & -1.69 & 1.70 & 0.29 \\
\hline 5 & 1.52 & -2.25 & 2.46 & 2.69 & -1.38 & -0.30 & -1.95 & 0.79 & -0.02 \\
\hline 6 & 1.64 & -2.59 & 2.65 & 3.50 & -1.99 & 0.59 & -2.13 & 0.18 & -0.27 \\
\hline \hline 11 & 1.94 & -3.21 & 2.98 & 5.61 & -3.22 & 2.55 & -2.80 & -1.33 & -0.87 \\
\hline 12 & 1.96 & -3.26 & 3.00 & 5.80 & -3.31 & 2.70 & -2.87 & -1.46 & -0.92 \\
\hline 13 & 1.98 & -3.29 & 3.02 & 5.94 & -3.38 & 2.81 & -2.91 & -1.56 & -0.96 \\
\hline 14 & 1.99 & -3.31 & 3.03 & 6.03 & -3.42 & 2.89 & -2.95 & -1.62 & -0.98 \\
\hline 15 & 2.00 & -3.32 & 3.04 & 6.10 & -3.45 & 2.94 & -2.98 & -1.67 & -1.00 \\
\hline
\end{tabular}

Table 2. Computed coefficients $\omega$ in different iterations.

We evaluate our algorithm on the publicly available colorto-gray benchmark dataset [2], where results of many other methods are available. Fig. 3 shows a few representative images. Our results, shown in the second column, preserve very well color contrast presented in the original images. For the images shown in the first, third and fifth rows, our results have quite different color orders compared with others. It bears out the fact that during decolorization, for neighboring pixels with similar brightness, color difference preservation and enhancement is very important. 

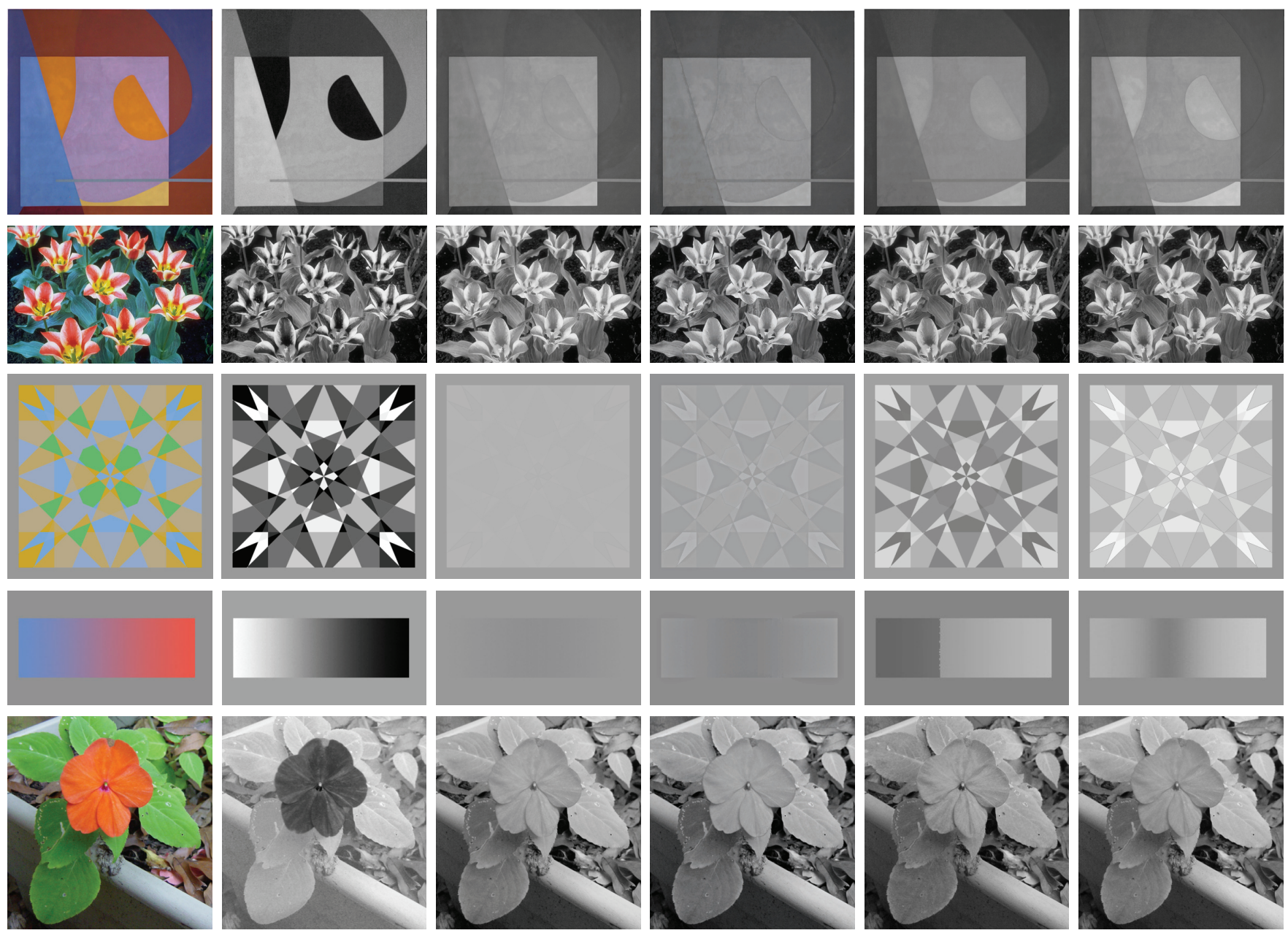

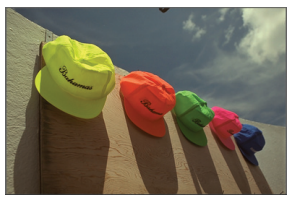

O L (

(a) Input

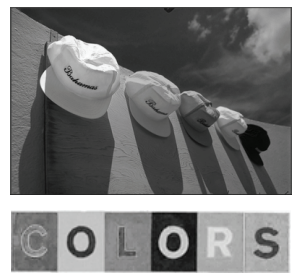

(b) Ours

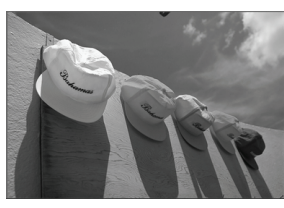

(c) $\mathrm{L}$ of $\mathrm{Lab}$

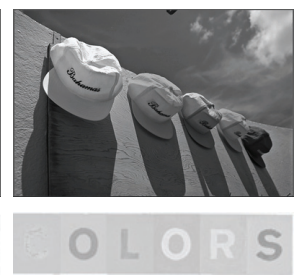

(d) Smith et al. [19]

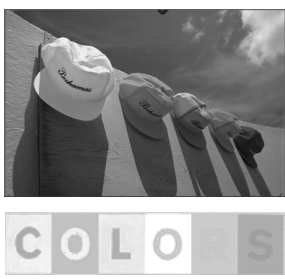

(e) Gooch et al. [6]

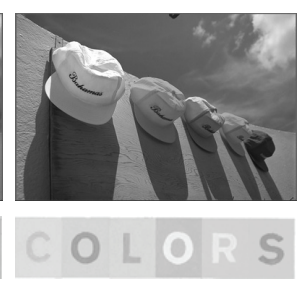

(f) Kim et al. [9]

Figure 3. Comparison with other decolorization methods.

Quantitative Evaluation To quantitatively evaluate the decolorization algorithms in terms of contrast preserving, we propose a new metric. It is based on the finding that if the color difference $\delta$ is smaller than a threshold $\tau$, it becomes nearly invisible in human vision. The task of contrast-preserving decolorization is therefore to maintain color change that is perceivable by humans. We define a color contrast preserving ratio (CCPR) as

$$
\mathrm{CCPR}=\frac{\#\left\{(x, y)|(x, y) \in \Omega,| g_{x}-g_{y} \mid \geq \tau\right\}}{\|\Omega\|},
$$

where $\Omega$ is the set containing all neighboring pixel pairs with their original color difference $\delta_{x, y} \geq \tau$. $\|\Omega\|$ is the

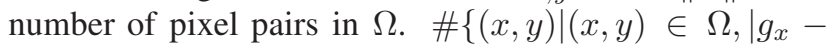
$\left.g_{y} \mid \geq \tau\right\}$ is the number of pixel pairs in $\Omega$ that are still distinctive after decolorization.

Based on CCPR, we quantitatively evaluate different methods using the 24 images in the dataset [2]. We calculate the average CCPR for the whole dataset by varying $\tau$ from 1 to $15^{2}$. Average CCPRs for other methods $[6,19,9]$

\footnotetext{
${ }^{2}$ It is suggested in [3] that color difference $\delta<6$ is generally imperceptible.
} 
are also collected. They are listed in Table 3. The quantities indicate that our method can preserve satisfactorily the color distinctiveness.

\begin{tabular}{|c||c|c|c|c|c|}
\hline$\tau$ & CIE & {$[19]$} & {$[6]$} & {$[9]$} & Ours \\
\hline \hline 1 & 0.66 & 0.70 & 0.69 & 0.72 & $\mathbf{0 . 7 6}$ \\
\hline 2 & 0.62 & 0.66 & 0.66 & 0.68 & $\mathbf{0 . 7 3}$ \\
\hline 3 & 0.61 & 0.64 & 0.64 & 0.67 & $\mathbf{0 . 7 2}$ \\
\hline 4 & 0.59 & 0.62 & 0.63 & 0.65 & $\mathbf{0 . 7 2}$ \\
\hline 5 & 0.59 & 0.61 & 0.63 & 0.64 & $\mathbf{0 . 7 2}$ \\
\hline 6 & 0.57 & 0.59 & 0.61 & 0.62 & $\mathbf{0 . 7 0}$ \\
\hline 7 & 0.56 & 0.58 & 0.60 & 0.60 & $\mathbf{0 . 6 9}$ \\
\hline 8 & 0.55 & 0.57 & 0.58 & 0.58 & $\mathbf{0 . 6 8}$ \\
\hline 9 & 0.54 & 0.56 & 0.57 & 0.57 & $\mathbf{0 . 6 7}$ \\
\hline 10 & 0.53 & 0.55 & 0.55 & 0.56 & $\mathbf{0 . 6 6}$ \\
\hline 11 & 0.52 & 0.54 & 0.54 & 0.54 & $\mathbf{0 . 6 5}$ \\
\hline 12 & 0.52 & 0.53 & 0.53 & 0.53 & $\mathbf{0 . 6 4}$ \\
\hline 13 & 0.51 & 0.52 & 0.52 & 0.52 & $\mathbf{0 . 6 3}$ \\
\hline 14 & 0.51 & 0.52 & 0.51 & 0.51 & $\mathbf{0 . 6 2}$ \\
\hline 15 & 0.50 & 0.51 & 0.50 & 0.50 & $\mathbf{0 . 6 1}$ \\
\hline
\end{tabular}

Table 3. Color contrast preserving ratio (CCPR) comparison.

More Results We show more natural image results in Fig. 4. The commonly used "rgb2gray" results are shown in (b) while ours are in (c). Note that the lightness channels in different color spaces, such as the L channel in "Lab", cannot preserve correct color contrast for many examples. When replacing these channels by our computed grayscale image, a contrast boosting effect can be yielded. As shown in Fig. 4(d), edges, patterns, and textures, after contract boosting, are getting more distinct.

\section{Concluding Remarks}

We have presented a new color-to-gray method that can well maintain or enhance the original color contrast. We leverage a weak color constraint to allow for very flexible and optimal grayscale representation, based on the fact that human perception has limited ability in determining order of color with respect to brightness. So rather than intuitively defining the sign of gray scale difference, we propose a mixture of Gaussian function to increase the search space in optimization. This strategy enables automatically finding suitable gray scales and preserves significant color change.

Note that people may have different feelings when seeing gray scales in different orders, as it is very subjective visual experience. It is thus necessary to provide mechanisms to flexibly manipulate color orders when users want to. We remark it is not a problem in our framework. Individual users can finely adjust the results produced by our method by adding their own color pairs that should satisfy specified orders to the constraint set.

\section{Acknowledgements}

The work described in this paper was supported by a grant from the Research Grants Council of the Hong Kong Special Administrative Region (Project No. 412911).

\section{References}

[1] R. Bala and R. Eschbach. Spatial color-to-grayscale transform preserving chrominance edge information. In Color Imaging Conference, pages 82-86, 2004.

[2] M. Cadík. Perceptual evaluation of color-to-grayscale image conversions. Computer Graphics Forum (Proc. Pacific Graphics 2008), 27(7):1745-1754, 2008.

[3] H. Chen and S. Wang. The use of visible color difference in the quantitative evaluation of color image segmentation. In Proceedings of International Conference on Acoustics, Speech, and Signal Processing (ICASSP), volume 3, pages 593-596, 2004.

[4] D. Corney, J. Haynes, G. Rees, R. Lotto, and O. Sporns. The brightness of colour. PLOS ONE, 4(3), 2009.

[5] M. Fairchild. Color appearance models. Wiley, 2005.

[6] A. A. Gooch, S. C. Olsen, J. Tumblin, and B. Gooch. Color2gray: salience-preserving color removal. ACM Transactions on Graphics (TOG), 24(3):634-639, 2005.

[7] M. Grundland and N. A. Dodgson. Decolorize: Fast, contrast enhancing, color to grayscale conversion. Pattern Recognition, 40(11):2891-2896, 2007.

[8] R. Hunter. Photoelectric color difference meter. Journal of the Optical Society of America, 48(12):985-993, 1958.

[9] Y. Kim, C. Jang, J. Demouth, and S. Lee. Robust color-togray via nonlinear global mapping. ACM Transactions on Graphics (TOG), 28(5), 2009.

[10] J. G. Kuk, J. H. Ahn, and N. I. Cho. A color to grayscale conversion considering local and global contrast. In Proceedings of Asian Conference on Computer Vision (ACCV), volume 4, pages 513-524, 2010.

[11] C. Lau, W. Heidrich, and R. Mantiuk. Cluster-based color space optimizations. In Proceedings of International Conference on Computer Vision (ICCV), pages 1172-1179, 2011.

[12] T. Lee, B. Kim, and W. Song. Converting color images to grayscale images by reducing dimensions. Optical Engineering, 49, 2010.

[13] R. Lotto and D. Purves. A rationale for the structure of color space. Trends in Neurosciences, 25(2):84-89, 2002.

[14] Y. Nayatani. Simple estimation methods for the Helmholtz Kohlrausch effect. Color Research and Application, 22(6):385-401, 1997.

[15] L. Neumann, M. Cadík, and A. Nemcsics. An efficient perception-based adaptive color to gray transformation. In Computational Aesthetics, pages 73-80, 2007.

[16] E. Ozgen. Language, learning, and color perception. Current Directions in Psychological Science, 13(3):95-98, 2004. 

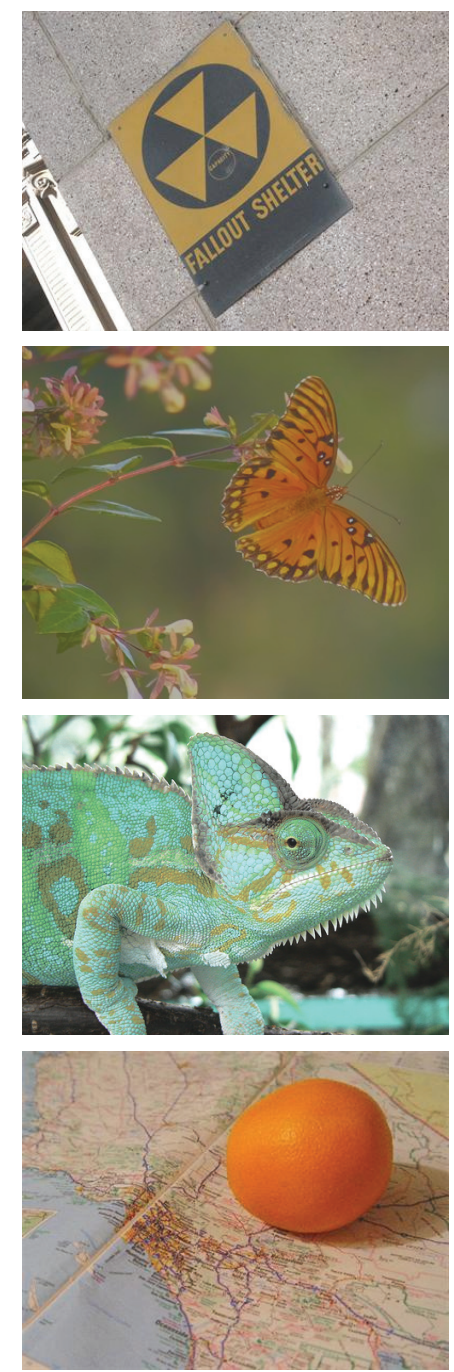

(a) Input
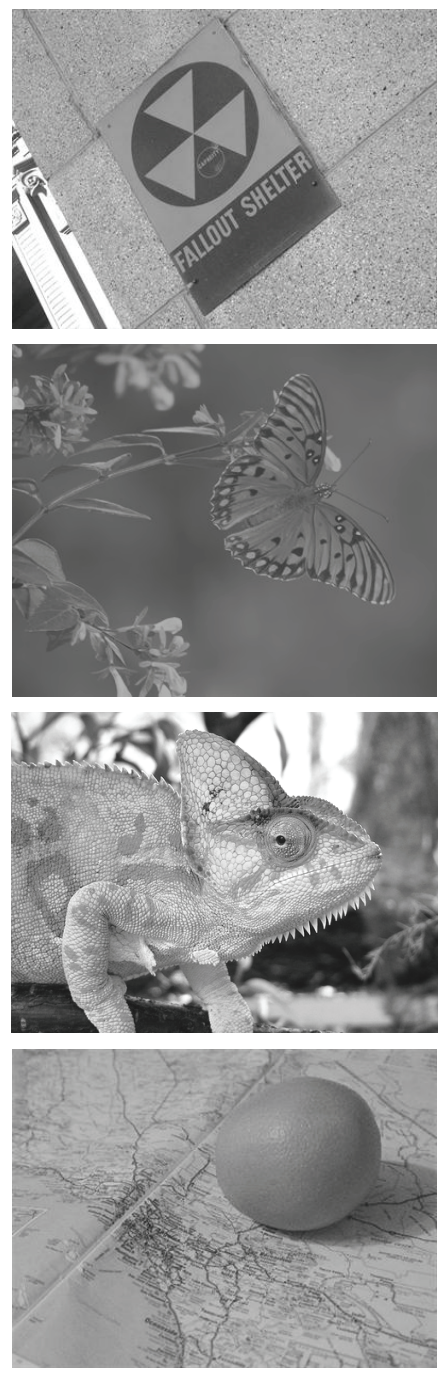

(b) rgb2gray()
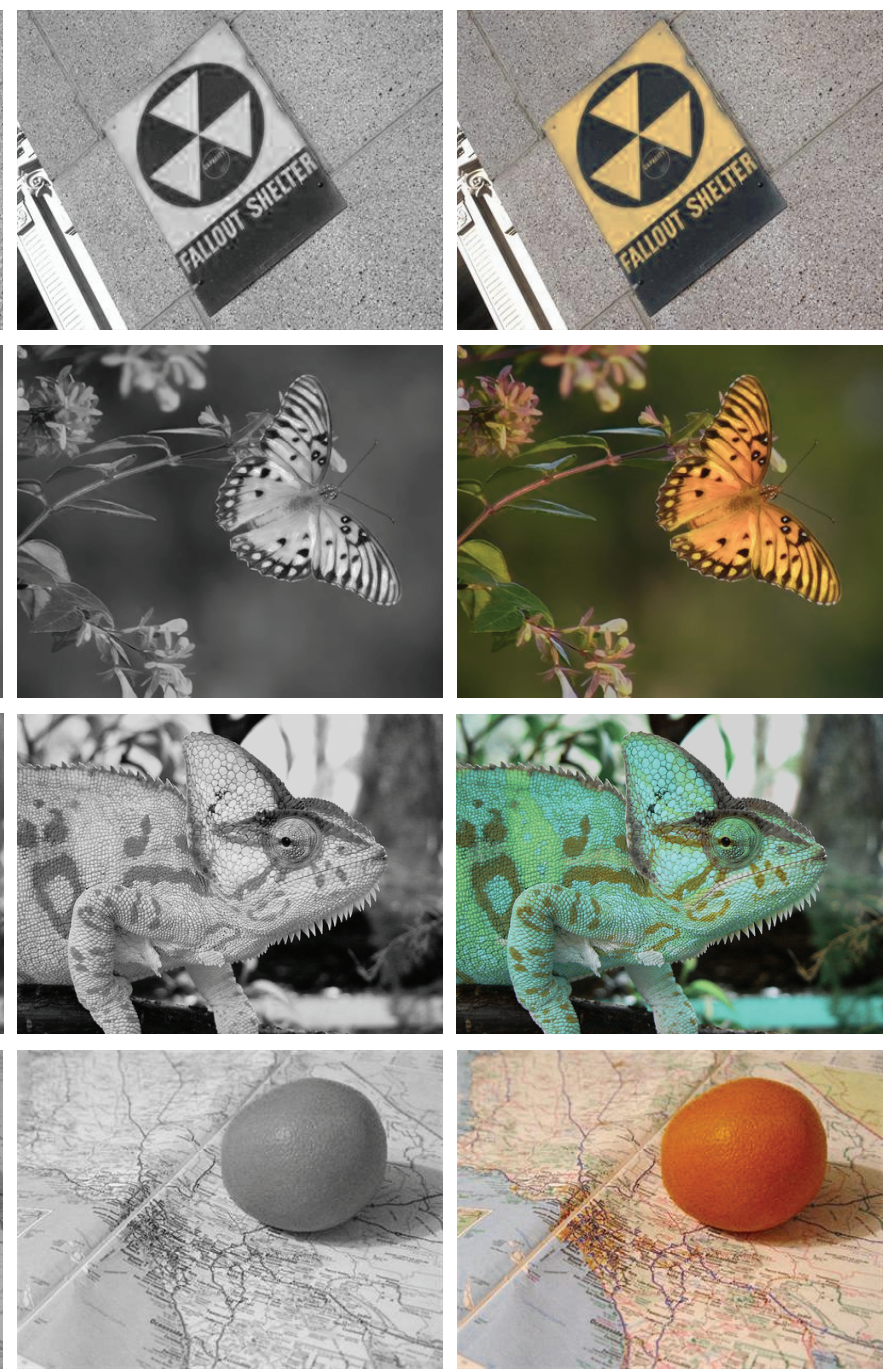

(c) Ours

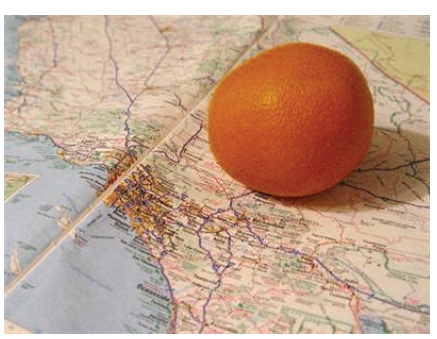

(d) Color Boosting

Figure 4. Natural image examples. Our grayscale image results can substitute the lightness channel in the Lab space to achieve contrast boosting, as shown in (d).

[17] K. Rasche, R. Geist, and J. Westall. Detail preserving reproduction of color images for monochromats and dichromats. IEEE Computer Graphics and Applications, 25(3):2230, 2005.

[18] G. Sharma and R. Bala. Digital Color Imaging Handbook. CRC Press, 2002.

[19] K. Smith, P. Landes, J. Thollot, and K. Myszkowski. Apparent greyscale: A simple and fast conversion to perceptually accurate images and video. Computer Graphics Forum (Proc. Eurographics 2008), 27(2):193-200, 2008.

[20] M. Song, D. Tao, C. Chen, X. Li, and C. W. Chen. Color to gray: Visual cue preservation. IEEE Transactions on Pattern Analysis and Machine Intelleligence., 32(9):1537-1552, 2010.

[21] B. Wong. Points of view: Color coding. Nature Methods, 7(8):573-573, 2010.
[22] G. Wyszecki and W. Stiles. Color Science: Concepts and Methods, Quantitative Data and Formulas. WileyInterscience, 2000.

[23] K. Zhou, L. Mo, P. Kay, V. Kwok, T. Ip, and L. Tan. Newly trained lexical categories produce lateralized categorical perception of color. In Proceedings of the National Academy of Sciences, number 22 in 107, pages 9974-9978, 2010. 